\title{
PRÁTICAS PARENTAIS PERCEBIDAS, AUTOESTIMA, OTIMISMO E RESILIÊNCIA EM FUTUROS PROFESSORES
}

\author{
Lidia Natalia Dobrianskyj Weber \\ UFPR \\ lidia@lidiaweber.com.br \\ Antoniela Yara Marques da Silva Dias \\ UFPR/FACEL \\ aymds@yahoo.com.br \\ Luana Cavicion Gomes \\ UFPR \\ luanacavicion@gmail.com \\ Rosana Angst Pasqualotto \\ UFPR \\ roangst@gmail.com
}

Fecha de Recepción: 25 Febrero 2018

Fecha de Admisión: 10 Abril 2018

\section{RESUMO}

As primeiras expectativas criadas pelos adolescentes acerca do seu futuro profissional são tomadas com base no contexto familiar. Esta pesquisa objetivou investigar relações entre as práticas educativas parentais percebidas e a escolha profissional, a autoestima, o otimismo e a resiliência em adolescentes que frequentam um curso técnico de formação de professores. Participaram 200 adolescentes, maioria do sexo feminino e idade média de 15,8 anos. Os instrumentos foram: questionário sociodemográfico, Escala de autoestima de Rosenberg, Life Orientation Test, Escala de Resiliência, Escalas de Qualidade na Interação Familiar. Os dados mostram que os jovens têm interesse em cursos universitários de licenciaturas e revelam identificação com a área do magistério. Sobre características boas de um professor, a maioria indicou comportamentos ligados à interação, seguido de cuidado com a profissão, e comportamentos relacionados ao domínio do conteúdo e didática. No caso dos comportamentos do professor a serem evitados, os mais citados foram o controle aversivo, a seguir foram comportamentos do professor que pudessem comprometer a aprendizagem, como a falta de planejamento e desorganização. Foram encontradas correlações significativas e positivas entre as práticas parentais percebidas pelos adolescentes e os escores de otimismo, resiliência e autoestima, revelando a importância do envolvimento dos pais para o desenvolvi- 


\section{PRÁTICAS PARENTAIS PERCEBIDAS, AUTOESTIMA, OTIMISMO E RESILIÊNCIA EM FUTUROS PROFESSORES}

mento de forças pessoais desses jovens que, desde cedo, definiram um objetivo de vida e estão no caminho para realizá-lo.

Palavras-chave: práticas educativas parentais; professor; autoestima; otimismo; resiliência

\section{ABSTRACT}

Perceived parenting practices, self-esteem, optimism and resilience in future teachers.

The first expectations created by adolescents regarding their professional future arise based on their family context. At this moment in life, adolescents decide about their future and make their decisions with the help of their families. The purpose of this study was to investigate relationships between perceived parenting practices and choice of profession, self-esteem, optimism and resilience in adolescents attending a technical teacher training course. Two hundred adolescents with an average age of 15.8 years took part in this study. The instruments used were: a sociodemographic questionnaire, the Rosenberg Self-Esteem scale, the Life Orientation Test, the Resilience Scale and the Family Interaction Quality Scales. The majority of students in this study are female, have few siblings and live with their parents. When asked about the university course they would like to take in the future, the absolute majority $(51 \%)$ chose degree courses providing teacher training. With regard to the reason why they chose to take a technical teacher training course, "making a dream come true" was the most frequent answer given by the respondents, followed by identification with this area. When asked about the characteristics of a good teacher, they mentioned dedication to the profession, dedication to students (involving aspects such as patience and affection). With regard to behaviours to be avoided by teachers, emphasis was placed on behaviours such as shouting, sarcasm, lack of teaching ability and arrogance. Significant and positive correlations were found between parenting practices perceived by the adolescents and scores for optimism, resilience and self-esteem, thus revealing the importance of parent involvement for the development of the personal strengths of these young people who, from an early age, defined an objective in life and are on their way to achieving it.

Keywords: parenting practices; teacher; self-esteem; optimism; resilience

\section{INTRODUÇÃO}

As primeiras expectativas criadas pelos adolescentes acerca do seu futuro profissional são tomadas com base no contexto familiar onde estão inseridos e nos modelos aprendidos. Neste momento de vida, os adolescentes decidem seu futuro e tomam decisões com 0 auxílio da sua família que tem a expectativa de um futuro e uma ascensão social melhor para os seus filhos (Martins, 2010; Inocêncio, 2013). Neste sentido, é importante destacar a importância e a influência dos estilos e das práticas educativas parentais para o desenvolvimento de crianças e adolescentes.

Baumrind $(1966,1968)$ descreve os três estilos. 0 estilo permissivo se caracteriza pela ausência de práticas punitivas a presença de aceitação e concordância com as ações da criança, com poucos pedidos em relação a tarefas de casa e mudança de comportamento. 0 pai com estilo permissivo evita se colocar como um agente responsável por modelar ou alterar o curso do comportamento da criança no futuro. 0 estilo autoritário é caracterizado pela tentativa de modelar, controlar e avaliar 0 comportamento da criança de acordo com um padrão absoluto de autoridade. 0 pai que exerce este estilo valoriza a obediência, faz uso da punição, restringe a autonomia e entende que a criança deve aceitar sua palavra como certa. Os pais com estilo autoritativo orientam a criança de uma maneira racional, incentivam-na a falar o que pensa, reconhecem seus interesses e suas qualidades, explicam as razões que regem determinadas regras, ouvem a criança quando esta não concorda com alguma regra estabelecida, bem como estabelecem padrões para comportamentos futuros. 
A partir deste modelo de estilos parentais de Baumrind, Maccoby e Martin (1983) propuseram duas dimensões a serem consideradas na caracterização de cada estilo parental, a exigência e a responsividade. A responsividade trata dos comportamentos dos pais que envolvem compreensão, comunicação e autonomia. Já a exigência refere-se a comportamentos dos pais que estão relacionados ao controle que exercem sobre os filhos (Costa, Teixeira \& Gomes, 2000). Esta nova proposição de Maccoby e Martin passa a classificar os estilos parentais com base em um índice entre responsividade e exigência: alta exigência e baixa responsividade são características do estilo parental autoritário, alta exigência e alta responsividade são características do estilo parental autoritativo, 0 estilo parental negligente caracteriza-se por baixa exigência e baixa responsividade, e 0 estilo parental indulgente/permissivo caracteriza-se pela alta responsividade e baixa exigência (Costa, Teixeira \& Gomes). Faria, Weber e Ton (2012) pesquisaram o estresse em vestibulandos e os dados revelaram mostrou que o primeiro fator que mais contribuiu para 0 estresse nesta população foram os estudos e, o segundo fator foi a família (24\%). Mais especificamente foi possível verificar correlação positiva entre 0 estresse dos vestibulandos com a instrusividade parental, enquanto que 0 apoio emocional dos pais teve correlação negativa com a apresentação de estresse.

Algumas pesquisas que estudam tratam a maneira de os pais educarem os filhos mostram a influência das práticas educativas em diversos aspectos do desenvolvimento de adolescentes. Weber (2017) realizou um estudo com o objetivo de investigar relações entre os estilos parentais percebidos e sinais de depressão, autoestima e comportamentos considerados antissociais em adolescentes. Com relação à autoestima, os resultados mostraram que os adolescentes que percebiam seus pais com estilo autoritativo eram aqueles que apresentavam autoestima elevada, já aqueles que apresentavam baixa autoestima e sinais de depressão eram os que percebiam seus pais como negligentes. No que se refere aos comportamentos antissociais, os adolescentes que relataram mentir sempre $(68 \%)$ e agredir outras pessoas $(50 \%)$ percebiam seus pais como negligentes, enquanto que a maioria $(40 \%)$ daqueles que relataram nunca praticar tal ação eram os que percebiam seus pais como autoritativos.

0 adolescente está em pleno processo de desenvolvimento e amadurecimento, e o modo como ele aprende por meio das suas interações com o mundo (seja a família, a escola, os professores, amigos) afetam a formação de sua autoestima. Guilhardi (2002) aponta que a autoestima é um sentimento produto de contingências de reforçamento positivo de origem social, ou seja, o adolescente ao relacionar-se constrói sua autoestima a partir de reforçadores sociais (atenção, carinho, afago físico, sorriso). No início de seus processos de aprendizagens relacionais, a família é o local onde a criança fica mais exposta, e deste modo é possível sugerir que os pais são determinantes iniciais para 0 desenvolvimento da autoestima das crianças e adolescentes. Deste modo, muitas crianças e adolescentes que vivenciam no seu dia a dia práticas educativas parentais inadequadas acabam tornando-se mais suscetíveis a construírem uma autoestima deficitária ao não se sentirem especiais e amadas (Weber, 2007).

Tantos jovens quanto adultos enfrentam adversidades, na vida e, portanto, é necessário que a família propicie estratégias para que haja o desenvolvimento de um repertório de comportamentos resilientes. 0 conceito de resiliência é definido como um processo interativo entre as pessoas e seu meio, o qual capacita e fortalece os indivíduos para lidar positivamente com as dificuldades. É válido ressaltar o caráter construtivo da resiliência, que não nasce com o indivíduo, mas é adquirido através de um processo de interação com o meio que vive (Lindstrom, 2001). No cotidiano das relações humanas, as pessoas passam por situações de crises e dificuldades, ficando mais vulneráveis, e suas conexões afetivas fragilizadas; nestes momentos são necessárias atitudes e capacidades relacionadas com a função de sustentar as relações e fortalecer os vínculos. (Assis, Pesce \& Avanci, 2006). 


\section{PRÁTICAS PARENTAIS PERCEBIDAS, AUTOESTIMA, OTIMISMO E RESILIÊNCIA EM FUTUROS PROFESSORES}

Uma forma de lidar com as adversidades é por meio do otimismo, que se caracteriza por um modo de olhar para a vida e avaliar o que os cerca de modo positivo e valorativo (Silva, 2011). Neste sentido, é relevante para o bem-estar psicológico das pessoas, e é definido pela tendência do indivíduo de acreditar em si e na sua capacidade de atingir seus objetivos (Fernández-González, González-Hernández \& Trianes-Torres, 2015).

Uma pesquisa realizada por Weber, Brandenburg e Viezzer (2003) revela que são os pais autoritativos que mais contribuem para o desenvolvimento do otimismo e que pais negligentes se relacionam a filhos mais pessimistas. Os indivíduos otimistas apresentam esperança e criam estratégias para solucionar os seus problemas (Snyder \& Lopez, 2009; Costa, 2017). 0 pensamento otimista possibilita criar e alcançar os objetivos almejados, favorece a visão do indivíduo face a acontecimentos futuros e torna-os possíveis de alcançar, além de amenizar situações de mal-estar e promover a saúde. 0 otimismo é um fator importante para as expectativas profissionais futuras de adolescentes. Adolescentes que possuem uma orientação positiva para a vida apresentam expectativas otimistas e objetivos concretizáveis, e ao contrário, jovens com uma orientação pessimista para a vida, revelam expectativas negativas, não têm objetivos claros e abdicam com facilidade de uma tarefa (Ottati, 2014).

Desta forma, o objetivo do presente trabalho foi investigar correlações entre as práticas educativas parentais, a escolha profissional, a autoestima, o otimismo e a resiliência em adolescentes que frequentam o curso técnico de Formaçãa de Professores.

\section{MÉTODO}

\section{Participantes:}

responderam a pesquisa 200 alunos do curso técnico de Formação de Professores localizado na região metropolitana de Curitiba, Brasil.

\section{Instrumentos:}

Questionário sociodemográfico para coletar dados como idade, gênero e contextos da família e perguntas relacionadas a sua futura prática profissional.

Escala de Autoestima de Rosenberg (Rosenberg, 1989 traduzida e adaptada por Hutz e Zanon, 2011): escala unidimensional que avalia um conjunto de sentimentos de autoestima e autoaceitação com 10 itens que são respondidos em uma escala likert de cinco pontos.

Life Orientation Test (LOT-R) (Scheier, Carver \& Bridge, 1995 traduzido e validado por Bastianello, Pacico \& Hutz, 2014): escala unidimensional que mede orientações otimistas ou pessimistas e é composta de 10 itens a serem respondidos em uma escala likert de cinco pontos.

Escala de Resiliência (Wagnild \& Young, 1993, traduzido por Pesce e cols., 2005): mede níveis de adaptação psicossocial positiva diante de eventos de vida importantes. Possui 25 itens descritos afirmativamente, com alternativas de resposta de acordo com escala do tipo likert de cinco pontos. Para a realização do presente trabalho a escala foi reduzida para 7 questões $(1,6,13,17,18,21$ e 23).

Escalas de Qualidade na Interação Familiar - EQIF (Weber, Prado, Salvador \& Brandenburg, 2008): avalia práticas educativas parentais e aspectos da interação familiar. Possui 40 itens, e a criança/adolescente responde as questões sobre práticas educativas parentais e vida familiar por meio de uma escala likert de cinco pontos. É dividida em nove escalas, sendo elas: envolvimento, regras e monitoria, comunicação positiva dos pais, comunicação negativa, punição corporal, clima conjugal positivo, clima conjugal negativo, modelo parental e sentimento dos filhos. 


\section{Procedimentos:}

a aplicação foi realizada coletivamente e cada estudante respondeu de modo individual e anônimo, sendo a participação voluntária e mediante a assinatura do Termo de Assentimento e Termo de Consentimento Livre e Esclarecido (TCLE).

\section{RESULTADOS E DISCUSSÃO}

Os 200 estudantes do curso técnico de Formação de Professores dividiram-se em 93\% do gênero feminino e $7 \%$ do gênero masculino, com idade média de 15,8 anos ( $D P=1,34)$. Quando perguntados sobre sua religião, 58\% se denominaram católicos, $25 \%$ evangélicos, $2 \%$ cristãos, $1 \%$ outras e 14\% estudantes não responderam ou não possuíam religião. 0 curso de Formação de Professores tem a duração de 4 anos, e a maioria dos participantes da pesquisa estavam no primeiro e segundo anos e relatam (Tabela 1) os motivos para a escolha do curso de professores.

Tabela 1: Alunos do curso de professores distribuídos percentualmente em relação ao motivo pelo qual escoIheram este curso

\begin{tabular}{ll}
\hline Motivo de escolha do curso & $\%$ \\
\hline Identificação com a área & $23,5 \%$ \\
Realização de um sonho & $16,7 \%$ \\
Gostar de crianças & $14,2 \%$ \\
Novas oportunidade & $10,0 \%$ \\
Transformação da realidade & $5,9 \%$ \\
Influência familiar & $6,8 \%$ \\
Outros motivos & $9,0 \%$ \\
Não responderam & $14,5 \%$ \\
\hline Total & $100,0 \%$ \\
\hline
\end{tabular}

A Tabela 1 mostra que grande parte dos respondentes afirmaram estar realizando o curso de formação de professores por compatibilidade com a área, seja em termos de competências técnicas (gostar de lecionar) ou relacionais (gostar de crianças). Apesar de menor porcentagem (10\%), também aparece a possibilidade do curso técnico proporcionar oportunidades futuras de trabalho ou experiência. Além disso, é expressiva a porcentagem de participantes que desejam continuar em cursos universitários relacionados à carreira docente - caso das licenciaturas $(52 \%)$ como matemática, biologia, letras, pedagogia etc., ou seja, desejam ser professores; em seguida gostariam de cursos na área da saúde (16\%), Ciências Humanas e Sociais (10\%), Artes (5\%) e outros ou não responderam (17\%). Vale destacar a complementaridade destes dados, uma vez que a escolha da faculdade futura parece ter relação com 0 motivo pelo qual estão realizando 0 atual curso, tal como a identificação com aspectos da vida docente.

A Tabela 2 revela quais comportamentos de um professor os participantes acham benéficos e prejudiciais 


\begin{tabular}{ll}
\hline COMPORTAMENTOS BENÉFICOS DO PROFESSOR & $\%$ \\
\hline Características relacionadas à interação & $49,0 \%$ \\
Dedicação à profissão & $22,7 \%$ \\
Domínio do conteúdo/didática & $18,0 \%$ \\
Domínio de turma/controle & $2,7 \%$ \\
Ética & $0,8 \%$ \\
Outros ou não responderam & $6,8 \%$ \\
\hline Total de comportamento benéficos do prof. & $100,0 \%$ \\
\hline COMPORTAMENTOS PREJUDICIAIS DO PROFESSOR & $\%$ \\
\hline Controle aversivo & $44,0 \%$ \\
Comportamentos que podem comprometer a aprendizagem & $15,0 \%$ \\
Desinteresse & $8,0 \%$ \\
Arrogância & $6,0 \%$ \\
Falar assuntos pessoais & $5,0 \%$ \\
Características pessoais que dificultam o andamento em sala de aula & $4,0 \%$ \\
Outros aspectos ou não responderam & $18,0 \%$ \\
\hline Total comportamentos prejudiciais do prof. & $100,0 \%$ \\
\hline
\end{tabular}

A Tabela 2 revela a importância dada aos relacionamentos em sala-de-aula, pois tanto a maior porcentagem de comportamentos considerados característicos de um bom professor (49\%) como aqueles a serem evitados (controle aversivo com 44\%), dizem respeito à interação professor-aluno. Outros aspectos considerados como positivos do professor diziam respeito à dedicação à profissão (23\%) e ao domínio do conteúdo e à didática (18\%). Dentre os aspectos secundários, mas também considerados prejudiciais na interação professor-aluno, podem-se destacar os comportamentos que pudessem comprometer a aprendizagem, como a falta de planejamento, desorganização, fornecer poucas oportunidades para 0 aluno se expressar (15\%), desinteresse (8\%), arrogância (6\%) e os comportamentos que possam dificultar 0 andamento em sala dela, tais como a irresponsabilidade $(4 \%)$.

Por meio das Escalas de Interação Familiar (EQIF) foram calculados os escores que classificam os jovens em situação de risco ou proteção familiar. Constatou-se que $23 \%$ estudantes se encontram em situação de risco, 24\% encontram-se em situação de proteção e 53\% estão em uma situação intermediária. Foram verificadas as relações entre os escores altos, médio e baixos de autoestima, resiliência, otimismo e estar em situação de risco e proteção por meio do teste de Qui-quadrado. Foi encontrada relação significativa entre altos níveis de autoestima e altos níveis de resiliência $\left(c^{2}=58,87, p<0,05\right)$. Foi também encontrada a clara relação entre autoestima e otimismo $\left(c^{2}=59,96\right.$, $p<0,05)$ e otimismo e resiliência $\left(c^{2}=78,93, p<0,05\right)$.

Foram encontradas correlações significativas entre as variáveis analisadas no estudo, sendo estas apresentadas na Tabela 3. 
Tabela 4: Correlações de Pearson das variáveis analisadas na presente pesquisa, sendo elas autoestima, otimismo, resiliência e EQIF

\begin{tabular}{|c|c|c|c|c|c|c|c|c|c|c|c|c|}
\hline & 1 & 2 & 3 & 4 & 5 & 6 & 7 & 8 & 9 & 10 & 11 & 12 \\
\hline 1. Autoestima de Rosenberg & 1 & $0,65^{* *}$ & $0,67^{* *}$ & $0,21 * *$ & $0,18^{*}$ & $0,23 * *$ & $-0,34 * *$ & $-0,21$ & 0,08 & $-0,17^{*}$ & $0,17^{*}$ & $0,21^{* *}$ \\
\hline 2. Otimismo (LOR-R) & $\begin{array}{l}0,65^{* *} \\
0,67 * *\end{array}$ & $\begin{array}{l}1 \\
0,67 * *\end{array}$ & $\begin{array}{l}0,67^{* *} \\
1\end{array}$ & $\begin{array}{l}0,28^{* *} \\
0,26 * *\end{array}$ & $\begin{array}{l}0,23 * * \\
0,22 * *\end{array}$ & $\begin{array}{l}0,16^{*} \\
0,22^{* *}\end{array}$ & $\begin{array}{l}-0,26 * * \\
-0,20 * *\end{array}$ & $\begin{array}{l}-0,17^{*} \\
-0,08\end{array}$ & $\begin{array}{l}0,18^{*} \\
0,16^{*}\end{array}$ & $\begin{array}{l}-0,18^{*} \\
-0,11\end{array}$ & $\begin{array}{l}0,22^{* *} \\
0,21^{* *}\end{array}$ & $\begin{array}{l}0,26^{* *} \\
0,21^{* *}\end{array}$ \\
\hline 3. Resiliência & $0,21^{* *}$ & $0,28^{* *}$ & $0,26^{* *}$ & 1 & $0,56^{* *}$ & $0,58^{* *}$ & $-0,56^{* *}$ & $-0,13$ & $0,51^{* *}$ & $-0,41^{* *}$ & $0,61^{* *}$ & $0,73 * *$ \\
\hline 4. Envolvimento Pai/Mãe & $0,18^{*}$ & $0,23 * *$ & $0,22 * *$ & $0,56^{* *}$ & 1 & $0,50^{* *}$ & $-0,33^{* *}$ & $-0,24 * *$ & $0,44 *$ & $-0,25^{*}$ & $0,60^{* *}$ & $0,61^{* *}$ \\
\hline 6. Comum Posit Pai/Mãe & $0,23 * *$ & $0,16^{*}$ & $0,22 *$ & $0,58^{* *}$ & $0,50^{* *}$ & 1 & $-0,40^{* *}$ & $-0,14^{*}$ & $0,35^{* *}$ & $-0,31^{* *}$ & $0,50^{* *}$ & $0,46^{* *}$ \\
\hline $\begin{array}{l}\text { 7. Comum Negat Pai/Mãe } \\
\text { 8. Punição Corporal Pai e Mãe }\end{array}$ & $\begin{array}{l}-0,34^{* *} \\
-0,21^{*}\end{array}$ & $\begin{array}{l}-0,26^{* *} \\
-0,17^{*}\end{array}$ & $-0,08$ & $-0,13$ & $-0,24 * *$ & $-0,14^{*}$ & $0,35^{* *}$ & $0,35 * *$ & 0,12 & 0,08 & $-0,11$ & $-0,19^{* *}$ \\
\hline 9. Clima conjugal positi Pai/mãe & 0,08 & $0,18^{*}$ & $0,16^{*}$ & $0,51^{* *}$ & $0,44^{* *}$ & $0,35^{* *}$ & $-0,28 * *$ & 0,12 & 1 & $-0,47^{* *}$ & $-0,58^{* *}$ & $0,56^{* *}$ \\
\hline $\begin{array}{l}\text { 10. Clima conjugal negati } \\
\text { Pai/Mãe }\end{array}$ & $-0,17^{*}$ & $-0,18^{*}$ & $-0,11$ & $-0,41^{* *}$ & $-0,25^{*}$ & $-0,31 * *$ & $0,63^{* *}$ & 0,08 & $-0,47^{* *}$ & 1 & $-0,44^{*}$ & $-0,45^{* *}$ \\
\hline $\begin{array}{l}\text { 11. Modelo Pai/mãe } \\
\text { 12. Sentimento Filho Pai/Mãe }\end{array}$ & $0,17^{*}$ & $\begin{array}{l}0,22 * * \\
0,26 * *\end{array}$ & $\begin{array}{l}0,21^{* *} \\
0,21^{* *}\end{array}$ & $0,61^{* *}$ & $\begin{array}{l}0,61 * * \\
0,61 * *\end{array}$ & $0,50^{* *}$ & $\begin{array}{l}-0,42 * * \\
-0,46 * *\end{array}$ & $\begin{array}{l}-0,11 \\
-0,19 * *\end{array}$ & $0,58^{* *}$ & $\begin{array}{l}-0,44^{*} \\
-0,45^{* *}\end{array}$ & $\begin{array}{l}1 \\
0,72 * *\end{array}$ & $\begin{array}{l}0,72^{* *} \\
1\end{array}$ \\
\hline
\end{tabular}

**Correlação com nível de significância a $0,01 *$ Correlação com nível de significância a 0,05

A Tabela 3 revela que há correlações significativas entre as práticas educativas parentais positivas (envolvimento do pai e da mãe, regras e monitoria e comunicação positiva) e fatores de proteção (autoestima, otimismo e resiliência). Esses dados evidenciam que, para se desenvolver uma boa autoestima, um bom nível de resiliência e otimismo, deve-se haver nas relações familiares afeto, noções de regras e limites e uma comunicação adequada.

De modo geral, verificou-se que, quanto mais expressivos os indicadores de comunicação e participação dos cônjuges em relação aos filhos, melhor o nível de otimismo. De acordo com Bolsoni \& Del Prette (2000) 0 envolvimento positivo dos pais na vida diária de seus filhos (participação nas atividades escolares, culturais e de lazer) contribuem para um desenvolvimento socioemocional saudável.

Esta Tabela 3 também mostra a correlação da comunicação positiva dos pais (pai e mãe juntos) com a autoestima, otimismo, resiliência, envolvimento dos pais, regras e monitoria do pai e da mãe, clima conjugal positivo, modelo parental e sentimento do filho em relação ao pai e à mãe. A comunicação positiva dos pais apresentou correlação negativa com a comunicação negativa, com a punição corporal ministrada pelos pais e com o clima conjugal negativo. Entendendo os dados, pode-se dizer que a correlação entre a comunicação positiva dos pais e a autoestima dos filhos vai ao encontro do estudo de Weber (2017) que evidencia as relações entre 0 estilo parental autoritativo (pais 
com este estilo tendem a apresentar comunicação positiva com seus filhos) e a autoestima. Ou seja, pais com estilo parental autoritativo possivelmente criam condições mais favoráveis para que seus filhos se avaliem de maneira mais positiva.

A comunicação positiva é uma variável essencial para a construção da autoestima, otimismo e resiliência. Constatou-se que quanto maior a comunicação positiva dos pais, melhor os filhos se avaliam (autoestima), percebem o mundo de maneira mais positiva (otimismo) e apresentam uma melhor capacidade de enfrentamento das dificuldades (resiliência dos filhos). Esses dados podem ser relacionados com os resultados apresentados na revisão sistemática realizada por Carvalho e Silva (2014), onde os autores mencionam um estudo de Garcia, Brino e Williams (2009) que indicou que dentre os fatores que dificultavam o desenvolvimento da resiliência estava a dificuldade de relacionamento com os pais. Em todos os casos parece ficar clara a relação existente entre as práticas parentais e o desenvolvimento da resiliência.

É também possível afirmar que quanto melhor é a comunicação positiva por parte dos pais, menor é 0 uso de punição - indicando assim, a comunicação como estratégia alternativa e protetiva ao uso da coerção. Da mesma forma, pode-se dizer que os pais que apresentam melhor comunicação positiva com os filhos também são aqueles que mantém um clima conjugal mais harmonioso, 0 que reafirma 0 valor da comunicação nas relações interpessoais. A partir destes dados fica evidente 0 quanto as práticas parentais são importantes variáveis protetivas a serem consideradas (Matsukura, e cols., 2013).

Há correlações entre os fatores de proteção otimismo e resiliência. Adolescentes que possuem uma orientação positiva para a vida apresentam expectativas otimistas e objetivos concretizáveis, porém adolescentes com uma orientação pessimista para a vida, apresentam expectativas negativas, não têm objetivos claros de vida e abdicam com facilidade de uma tarefa (Ottati, 2014). Também foram encontradas correlações positivas entre o modelo parental e a autoestima, a resiliência, e o otimismo, evidenciando que quando os filhos percebem coerência entre 0 dizer e o fazer de seus pais (modelo), melhor é a sua autoavaliação, enfrentam adversidades e pensam mais positivamente. Os pais devem apresentar modelos comportamentais, os quais têm um papel importante para o desenvolvimento crianças e adolescentes (Weber, 2007).

No que se refere ao sentimento dos filhos em relação aos pais, esta variável correlacionou-se positivamente com a autoestima, o otimismo, a resiliência, o envolvimento dos pais, comunicação positiva e modelo parental. Em contrapartida, correlacionou-se negativamente, com as regras e monitoria, com a comunicação negativa dos pais, com a punição corporal e 0 clima conjugal negativo. Infelizmente, pesquisas revelam maioria das famílias utiliza ou já utilizou essa prática coercitiva da punição corporal no Brasil (Weber \& Weber, 2014). Esses dados mostram que quando um filho se sente afetivamente ligado a seus pais melhor ele se avalia, e percebe os pais com boa comunicação e como um modelo de vida.

Da mesma forma que as práticas educativas parentais positivas favorecem a aprendizagem de um repertório socialmente competente, as práticas educativas parentais negativas, prejudicam 0 aprendizado de um repertório rico em habilidades sociais. No caso da presente pesquisa, a punição corporal teve correlação negativa com a autoestima. A punição produz efeitos colaterais indesejados e não possibilita a construção da autoconfiança necessária para sua vida futura.

De acordo com os dados do presente estudo foram encontradas correlações entre as variáveis relacionadas às práticas parentais, otimismo, resiliência e autoestima. Foi possível verificar a importância do envolvimento dos pais e a prática do estilo autoritativo para o desenvolvimento adequado da autoestima, otimismo e resiliência desses adolescentes que têm o desejo de seguir uma carreira como professor. 
Uma limitação do estudo refere-se à exclusiva medida de autorrelato, entretanto, o presente trabalho não pretende esgotar a temática trabalhada, mas instiga à realização de mais pesquisas para compreender quais variáveis podem auxiliar no desenvolvimento da resiliência, otimismo e autoestima em adolescentes, utilizando, além de medidas indiretas, medidas diretas do comportamento.

\section{REFERÊNCIAS}

Assis, S.G., Pesce, R.P. \& Avanci, J.Q. (2006). Resiliência: enfatizando a proteção dos adolescentes. Porto Alegre: Artmed.

Bastianello, M.R., Pacico, J.C. \& Hutz, C.S. (2014). Optimism, self-esteem and personality: adaptation and validation of the brazilian version of the Revised Life Orientation Test (LOT-R). PsicoUSF, 19(3), 523-531, 2014.

Baumrind, D. (1966). Effects of authoritative control in child behavior. Child Development, 37, 888907.

Baumrind, D. (1968). Authoritarian vs. Authoritative parental control. Adolescence, 3(11), 255-272.

Bolsoni-Silva, A.T., Del Prette, A. \& Del Prette, Z.A.P. (2000). Relacionamento pais filhos: um programa de desenvolvimento interpessoal em grupo. Psicologia Escolar e Educacional, 3(3), 203215

Carvalho, M.S.D.P. \& Silva, B.M.B. (2014). Estilos parentais: um estudo de revisão bibliográfica. Revista Psicologia em Foco, 6(8), 22-42.

Costa, A.C.M. (2017). Autoconceito, otimismo e expectativas profissionais em adolescentes do ensino profissional e do ensino secundário. Dissertação de Mestrado, Psicologia da Educação, Universidade de Trás-os-Montes e Alto Douro, Portugal.

Costa, F.T., Teixeira, M.A.P. \& Gomes, W. B. (2000). Responsividade e exigência: duas escalas para avaliar estilos parentais. Psicologia: Reflexão e Crítica, 13(3), 465-473.

Faria, R.R., Weber, L.N.D. \& Ton, C.T. (2012). 0 estresse entre vestibulandos e suas relações com a família e a escolha profissional. Psicologia Argumento, 30(68), 43-52.

Fernández-González, L., González-Hernández, A. \& Trianes-Torres, M. (2015). Relationships between academic stress, social support, optimism-pessimism and self-esteem in college students. Electronic Journal of Research in Educational Psychology, 13(1), 111-130.

Guilhardi, H. J. (2002). Autoestima, autoconfiança e responsabilidade. Em: M. Z. S. Brandão e cols. Comportamento humano: tudo ou quase tudo que você queria saber para viver melhor (pp. 6398). Santo André: Esetec.

Inocêncio, P. (2013). O otimismo e as preocupações de carreira de adultos, desempregados ou empregados em processo de formação. Dissertação de Mestrado em Psicologia da Educação. Universidade do Algarve, Portugal.

Hutz, C. S. \& Zanon, C. (2011). Revisão da adaptação, validação e normatização da escala de autoestima de Rosenberg. Avaliação psicológica, 10(1), 41-49.

Lindstrom, B. (2001). 0 significado da resiliência. Adolescência latino-americana, 2(3), 133-137.

Matsukura, T.S., Cid, M.F.B., Angelucci, T.C. \& Minatel, M.M. (2013). Situações estressoras e fatores protetivos: percepções de meninas adolescentes que cumprem medidas socioeducativas. 0 Mundo da Saúde, 37(1), 25-34.

Martins, A. (2010). Preocupações de carreira e adaptabilidade: Um estudo exploratório numa amostra de candidatos a sargentos e oficiais de exército português. Dissertação de Mestrado. Faculdade de Psicologia e de Ciências da Educação, Lisboa, Portugal.

Maccoby, E. \& Martin, J. (1983). Socialization in the context of the Family: parent-child interaction. In E.M. Hetherington \& P.H. Mussen (Orgs.), Handbook of the child psychology Vol 4. 
Socialization, personality and the development (pp. 1-101). New York: Wiley.

Ottati, F. (2014). Otimismo, interesses profissionais e auto eficácia: Estudo com adolescentes. Tese de Doutorado. Universidade São Francisco, Brasil.

Pesce, R.P., Assis, S.G., Avanci, J.Q., Santos, N., Malaquias, J.V. \& Carvalhaes, R. (2005). Adaptação transcultural, confiabilidade e validade da escala de resiliência. Cadernos de Saúde Pública, 21(2), 436-448.

Rocha, G.V.M., Ingberman, Y.K. \& Breus, B. (2011) Análise da relação entre práticas parentais e 0 autoconceito de pré-escolares. Revista Brasileira de Terapia Comportamental e Cognitiva, XII(1), 87-106.

Silva, S. (2011). Pedagogia do Ressentimento: 0 otimismo nas concepções e nas práticas de ensino. Revista Brasileira Estudos Pedagógicos, 92(230), 107-125.

Snyder, C. R. \& Lopez, S. J. (2009). Psicologia positiva: uma abordagem científica e prática das qualidades humanas. Porto Alegre: Artmed.

Weber, L.N.D., Prado, P.M., Salvador, A.P.V. \& Brandenburg, O. J. (2008). Construção e confiabilidade das escalas de qualidade na interação familiar. Psicologia argumento, 26(52), 55-65.

Weber, L. N. D. (2017). Eduque com carinho: equilíbrio entre amor e limites (6

a ed.). Curitiba: Juruá.

Weber, L. N. D. (2017). Relações entre práticas educativas parentais percebidas e a autoestima, sinais de depressão e 0 uso de substâncias por adolescentes. International Journal of Developmental and Educational Psychology, 2(1), 157-168.

Weber, L.N.D., Brandenburg, O.J. \& Viezzer, A.P. (2003). A relação entre o estilo parental e 0 otimismo da criança. Psico-USF, 8(1), 71-79.

Weber, L.N.D. \& Weber, T.D. (2014). Disciplina coercitiva: relato de estudantes universitários brasileiros. International Journal of Developmental and Educational Psychology/INFAD, 2, 467-474. 